\title{
7 nm Spatial Resolution in Soft X-ray Microscopy
}

Benedikt Rösner, ${ }^{1 *}$ Frieder Koch, ${ }^{1}$ Florian Döring, ${ }^{1}$ Vitaliy A. Guzenko, ${ }^{1}$ Markus Meyer, ${ }^{2}$ Joshua L. Ornelas, ${ }^{2}$ Andreas Späth, ${ }^{2}$ Rainer H. Fink, ${ }^{2}$ Stefan Stanescu, ${ }^{3}$ Sufal Swaraj, ${ }^{3}$ Rachid Belkhou, ${ }^{3}$ Benjamin Watts, ${ }^{1}$ Jörg Raabe, ${ }^{1}$ Christian David ${ }^{1}$

${ }^{1 .}$ Paul Scherrer Institut, 5232 Villigen PSI, Switzerland

2. Department Chemistry and Pharmacy, FAU Erlangen-Nürnberg, Egerlandstr. 3, 91058 Erlangen, Germany

${ }^{3 .}$ Synchrotron SOLEIL, L'ormes des Merisiers, Saint Aubin BP-48, 91192, Gif-Sur-Yvette Cedex, France

* Benedikt Rösner, benedikt.roesner@psi.ch

X-ray microscopy and spectroscopy are powerful tools to gain valuable insight into many exciting, scientifically relevant samples. Development in microscopic methods has always aimed for achieving spatial resolutions as high as possible. Although highly confined X-ray beams with spot sizes well below $10 \mathrm{~nm}$ have been characterized in recent years using mirrors [1], Fresnel zone plate (FZP) lenses [2, 3] or multilayer Laue lenses [4, 5], X-ray imaging with single-digit nanometer resolution remains an extreme challenge.

We report on unprecedented spatial resolution down to $7 \mathrm{~nm}$ achieved at two soft X-ray microscopes using photon energies between $700 \mathrm{eV}$ and $850 \mathrm{eV}$. The experiments were performed at the scanning transmission X-ray microscopes (STXM) at the PolLux beamline at the Swiss Light Source [6] and the Hermes beamline at Synchrotron Soleil [7]. The scientific applications of these two instruments lie in the combination of microscopy and spectroscopy, where high spatial resolution provides a major benefit for scientific research.

The key element in a STXM for achieving small spot sizes and thus high resolution is the Fresnel zone plate (FZP) commonly used as X-ray lens. An elegant method to fabricate high-resolution FZPs has been introduced by doubling the line density obtained from the lithography step utilizing atomic layer deposition [8,9]. This method has been applied to fabricate FZPs with zone widths down to $12 \mathrm{~nm}$ [8-10]. By further optimization of the nanofabrication process, we have fabricated FZPs with outermost zone widths well below $10 \mathrm{~nm}$ and heights between $60 \mathrm{~nm}$ and $80 \mathrm{~nm}$ [11]. The specifications for the lenses were chosen according to the illumination at the PolLux and Hermes beamlines [6, 7], ensuring that the zone plate dimensions match the coherence length and energy bandwidth of each beamline. To achieve the highest possible spatial resolution, the experimental geometry and mechanical stability of the STXM endstations had to be optimized. Special attention has to be paid to the extremely short focal length, and to the mechanical stability of the sample stage. While the former challenge can be addressed by using a thin order-sorting aperture, the latter one has to be addressed by carefully optimizing the position control and feedback system. In this way, the maximum amplitude of sample displacement with respect to the zone plate has been brought to $\pm 3 \mathrm{~nm}$ perpendicular to the beam axis.

In order to characterize the spatial resolution of our FZPs, we recorded images of high-resolution test patterns. The test patterns consist of a periodic repetitions of an iridium line, an HSQ line, a second iridium line, and a gap. The lateral dimensions of each line or gap of the investigated test samples are $10 \mathrm{~nm}, 9 \mathrm{~nm}, 8 \mathrm{~nm}$, and $7 \mathrm{~nm}$ (Figure 1). We scanned the test objects in point-by-point mode with steps 


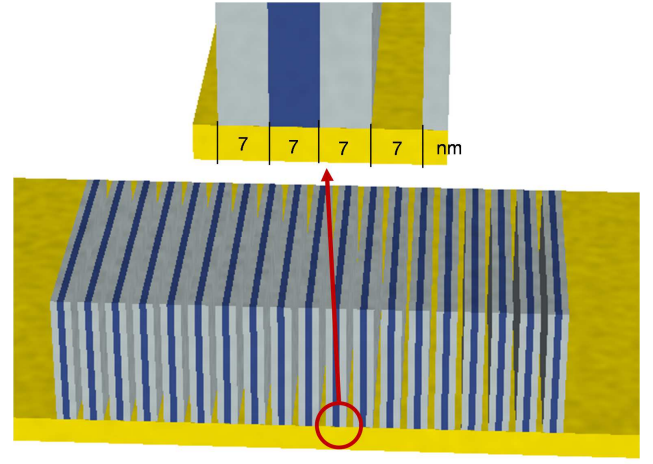

Figure. 1. Illustration of the $7 \mathrm{~nm}$ test sample for characterization of the spatial resolution.
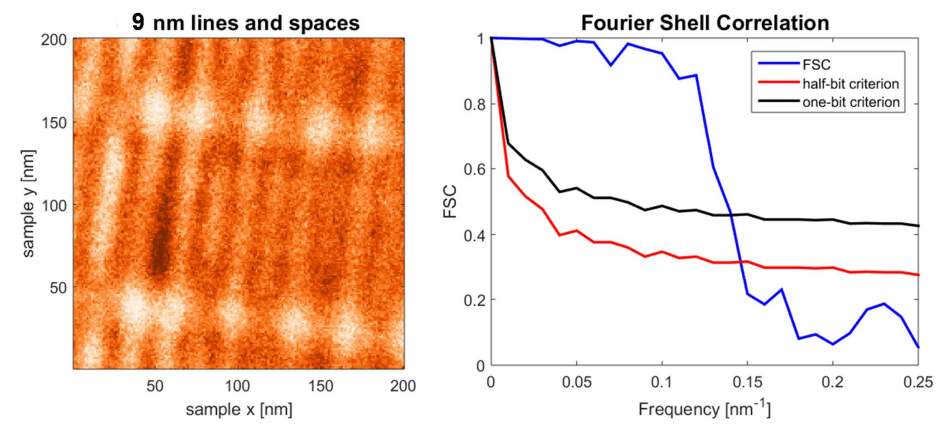

Figure. 2. a) High resolution $X$-ray micrograph of the test sample with $9 \mathrm{~nm}$ iridium lines and spaces, recorded at the Pollux STXM at $700 \mathrm{eV}$. b) Fourier shell correlation of a). of one nanometer. Figure 2a shows a STXM micrograph of a test pattern with $9 \mathrm{~nm}$ lines and spaces recorded at the Pollux STXM at $700 \mathrm{eV}$. To further reduce the noise, we saved the average position of each recorded pixel from the interferometer feedback loop and interpolated the recorded intensity to a regular grid. Determination of the spatial resolution was conducted using a twodimensional Fourier shell correlation [2, 12] of two independent data sets. This yields a frequency cut-off at $0.15 \mathrm{~nm}^{-1}$ in Fourier space, or $6.8 \mathrm{~nm}(7.1 \mathrm{~nm})$ in real space considering the half-bit (one bit) criterion (Figure 2b).

Whereas the Pollux beamline is limited by the coherence of the radiation from the bending magnet, the Hermes beamline provides higher flux at conditions for diffraction-limited imaging. Figure 3 shows the raw, as recorded image of a $7 \mathrm{~nm}$ test pattern without further post-processing or filtering. The achieved spatial resolution sets a new benchmark in X-ray microscopy.

\section{References:}

[1] Mimura H, Handa S, Kimura T et al, Nat Phys 6 (2010), p. 122.

[2] Mohacsi I, Vartiainen I, Rösner B et al, Sci Rep 7 (2017), p. 43624.

[3] Döring F, Robisch A L, Eberl C et al, Opt Express 21 (2013), p. 19311.

[4] Morgan A J, Prasciolu M, Andrejczuk A et al, Sci Rep 5 (2015), p. 9892.

[5] Bajt S, Prasciolu M, Fleckenstein H et al, Light-Sci Appl accepted (2017), p.

[6] Raabe J, Tzvetkov G, Flechsig U et al, Rev Sci Instrum 79 (2008), p. 113704.

[7] Belkhou R, Stanescu S, Swaraj S et al, J Synchrotron Rad 22 (2015), p. 968.

[8] Jefimovs K, Vila-Comamala J, Pilvi T et al, Phys Rev Lett 99 (2007), p. 264801.

[9] Vila-Comamala J, Jefimovs K, Raabe J et al, Ultramicroscopy 109 (2009), p. 1360.

[10] Vila-Comamala J, Gorelick S, Färm E et al, Opt Express 19 (2011), p. 175.

[11] Rösner B, Koch F, Döring F et al, Microelectron Eng 191 (2018), p. 91.

[12] van Heel M and Schatz M, J Struct Biol 151 (2005), p. 250. 